\title{
Surgical Decision-Making in Multivalvular Heart Disease: A Holistic Approach
}

\author{
Neeti Makhija $\quad$ Rohit Malhotra ${ }^{1}$ Rohan Magoon² \\ ${ }^{1}$ Department of Cardiac Anaesthesia, Cardiothoracic Centre, \\ CNC, All India Institute of Medical Sciences, New Delhi, India \\ ${ }^{2}$ Department of Cardiac Anaesthesia, Atal Bihari Vajpayee Institute \\ of Medical Sciences (ABVIMS) and Dr. Ram Manohar Lohia Hospital, \\ New Delhi, India \\ ${ }^{3}$ Department of Cardiothoracic and Vascular Surgery, \\ Cardiothoracic Centre, CNC, All India Institute of Medical Sciences, \\ New Delhi, India \\ J Card Crit Care:2020;4:66-69
}

\begin{abstract}
Address for correspondence Neeti Makhija, MD, Department of Cardiac Anaesthesia, Cardiothoracic Centre, All India Institute of Medical Sciences, Room No. 9, 7th Floor, Ansari Nagar, New Delhi-110029, India (e-mail: neetimakhija@hotmail.com).
\end{abstract}

\author{
Abstract \\ Keywords \\ - antiretroviral therapy \\ - multivalvular disease \\ - repair versus \\ replacement \\ - anticoagulation
}

Patients with multivalvular heart disease often presents a unique challenge with regard to the intraoperative decision-making in the form of choosing between a satisfactory valvular repair or a more definitive valve replacement. We describe a patient with multivalve involvement on antiretroviral therapy. Discussion presents a nuanced perspective on surgical decision-making in multi-valvular heart disease, wherein an effective communication between the surgeon and the echocardiographer is required for ensuring favorable postoperative outcomes.

\section{Introduction}

The cohort of patients with multivalvular heart disease often presents a unique challenge with regard to the intraoperative decision-making in the form of choosing between a satisfactory valvular repair and a more definitive valve replacement. The scenario is often compounded by practicalities such as age of the patient, planned future pregnancy in females, life expectancy, comorbid profile, surgical expertise, postoperative anticoagulation targets, and interactions with concomitant drug therapy.

\section{Case Report}

We describe a case of 35-year-old male, a known case of rheumatic heart disease on antiretroviral therapy (ART), scheduled for mitral valve repair/replacement. During preoperative evaluation, two-dimensional (2D) transthoracic echocardiography was suggestive of moderate-to-severe mitral regurgitation (MR) with mild mitral stenosis (MS), trivial tricuspid regurgitation (TR), and mild aortic regurgitation (AR). Left heart chambers were enlarged with normal biventricular function. Following routine preanesthetic evaluation and preparation in the preoperative period patient was wheeled into operation theater.

Induction of anesthesia was done according to standard protocol. Transesophageal echocardiography (TEE) evaluation in the pre-CPB period (-Video 1) was suggestive of moderate-to-severe MR ( - Fig. 1), moderate MS (-Fig. 2), moderate AR ( - Fig. $\mathbf{3}$ ), and TR. The mitral valve TEE assessment revealed thickening and restriction in movement of posterior mitral leaflet (PML) and prolapse of the anterior mitral leaflet (AML), most likely due to $\mathrm{A} 2$ prolapse, leading to

\section{Video 1}

Two-dimensional midesophageal long axis color compare transesophageal echocardiographic view at 121 degrees, showing moderate-to-severe mitral regurgitation with anterior mitral leaflet prolapse and moderate-to-severe aortic regurgitation secondary to right coronary cusp prolapsed. Online content including video sequences viewable at: https://www.thieme-connect.com/products/ejournals/ html/10.1055/s-0040-1713546.
DOI https://doi.org/

10.1055/s-0040-1713546

ISSN 2457-0206.
(C2020 Official Publication of The Simulation Society (TSS), accredited by International Society of Cardiovascular Ultrasound (ISCU).
License terms

() (1) $\ominus \circledast$ 


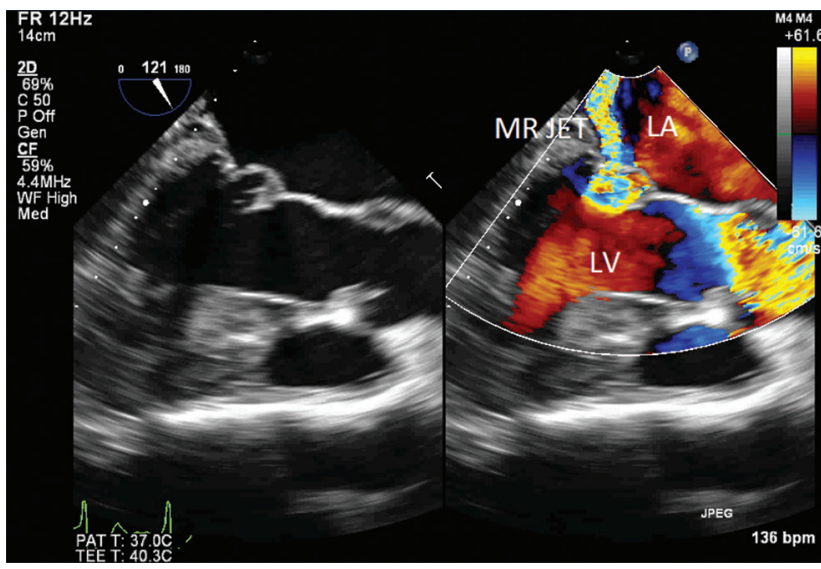

Fig. 1 Two-dimensional midesophageal long axis color compare transesophageal echocardiographic view at 121 degrees, showing eccentric mitral regurgitant jet with anterior mitral leaflet prolapse. Abbreviations: LA, left atrium; LV, left ventricle; MR, mitral regurgitation.

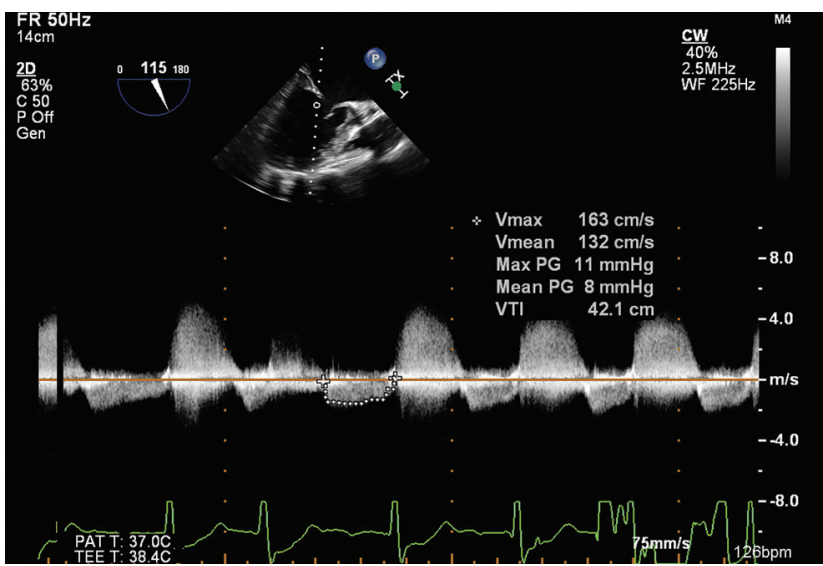

Fig. 2 Two-dimensional midesophageal long axis transesophageal echocardiographic view at 115 degrees, showing a mean pressure gradient of $8 \mathrm{~mm} \mathrm{Hg}$ across the mitral valve.

eccentric jet. The gradient across mitral valve was $8 \mathrm{~mm} \mathrm{Hg}$ ( - Fig. 2). Examination of the aortic valve revealed prolapse of right coronary cusp (RCC) ( - Fig. 4), leading to eccentric regurgitant jet (-Fig. 5).

After initiation of cardiopulmonary bypass (CPB) and postcardioplegic arrest, mitral valve was examined to have thickened and fixed PML. At A2-A3 junction, tethered secondary chordae led to improper coaptation, which appeared as A2 pseudoprolapse, with A3 actually going down due to retraction. Mitral valve commissures were fused and there was annular dilatation. Peroperative examination of the aortic valve revealed RCC large and prolapsing, and noncoronary cusp (NCC) retracted with dilated aortic valve annulus.

Mitral valve repair was attempted by division of tethered chordae, PML augmentation with fresh autologous pericardial patch, and customized teflon collar posterior ring annuloplasty size 31 . Aortic valve repair was done with

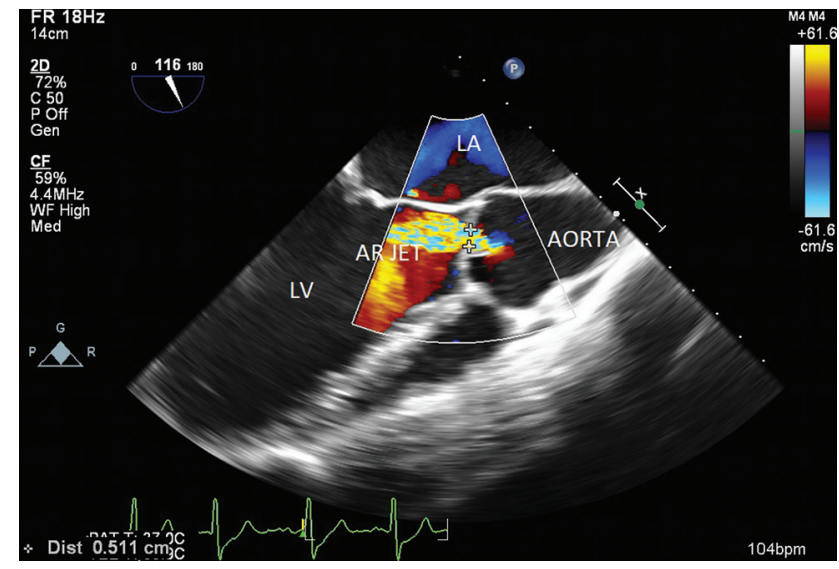

Fig. 3 Two-dimensional midesophageal aortic valve long axis transesophageal echocardiographic view at 116 degrees, showing aortic valve regurgitation with a vena contracta of $0.51 \mathrm{~cm}$. Abbreviations: $\mathrm{LA}$, left atrium; LV; left ventricle; AR, aortic regurgitation.

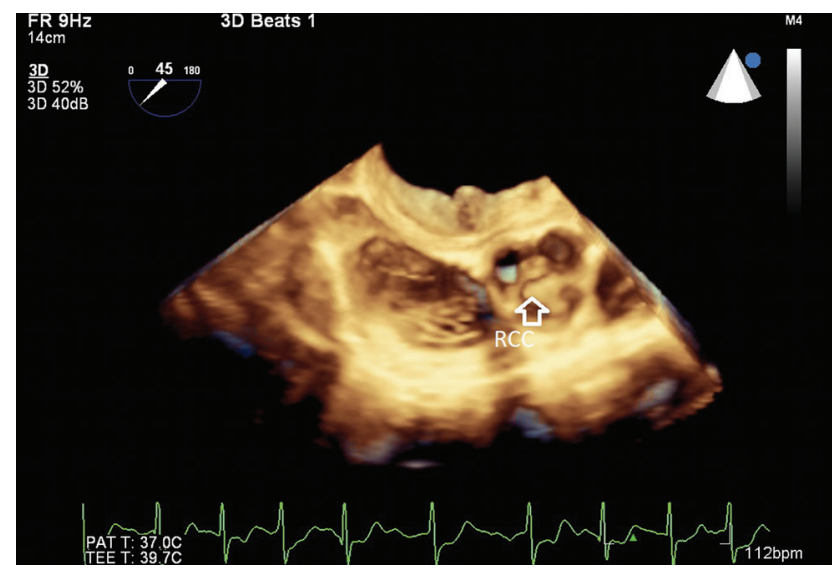

Fig. 4 Three-dimensional midesophageal aortic valve short axis transesophageal echocardiographic view, showing prolapse of RCC. Abbreviation: RCC, right coronary cusp.

subcommissural annuloplasty and plication of RCC prolapse. Weaning from $\mathrm{CPB}$ was done after adequate rewarming. PostCPB TEE evaluation revealed mean gradient of $6.0 \mathrm{~mm} \mathrm{Hg}$ (-Fig. 6) across mitral valve, suggestive of moderate mitral valve stenosis, and moderate-to-severe aortic regurgitation ( -Fig. 7). There was no residual mitral regurgitation. CPB was restarted and aortic valve replacement with St. Jude's Medical Regent size 19, a mechanical prosthetic valve, was done. Mitral valve was tackled with anterolateral and posteromedial commissurotomy. Weaning from $\mathrm{CPB}$ after adequate rewarming was done. Post-CPB TEE assessment of mitral valve revealed a trivial regurgitant jet with a mean gradient of $5 \mathrm{~mm} \mathrm{Hg}$ ( - Fig. 8). Aortic valve assessment showed normal functioning prosthetic valve with normal gradients. After stabilization of hemodynamics, patient was shifted to ICU for postoperative care. The postoperative phase was uneventful. 


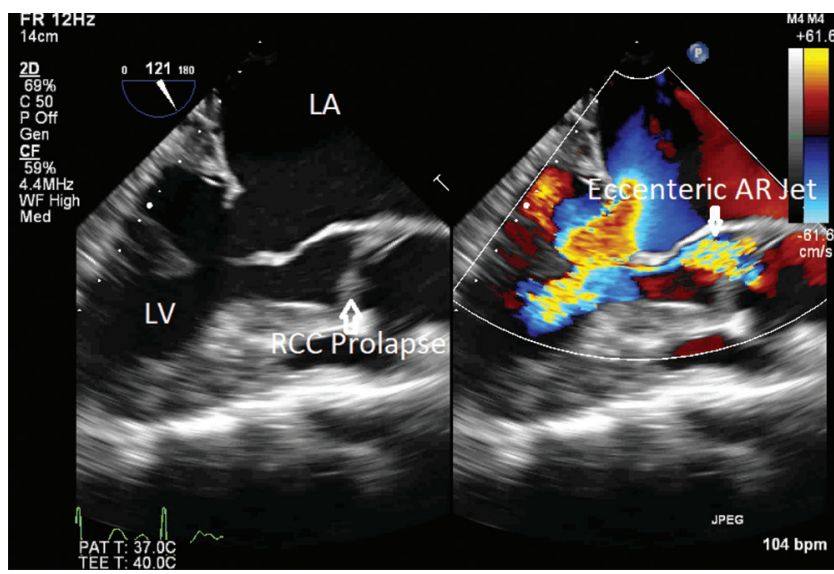

Fig. 5 Two-dimensional midesophageal aortic valve long axis transesophageal echocardiographic view at 121 degrees, showing aortic valve eccentric regurgitation jet with RCC prolapse. Abbreviations: LA, left atrium; LV, left ventricle; AR, aortic regurgitation; RCC, right coronary cusp.

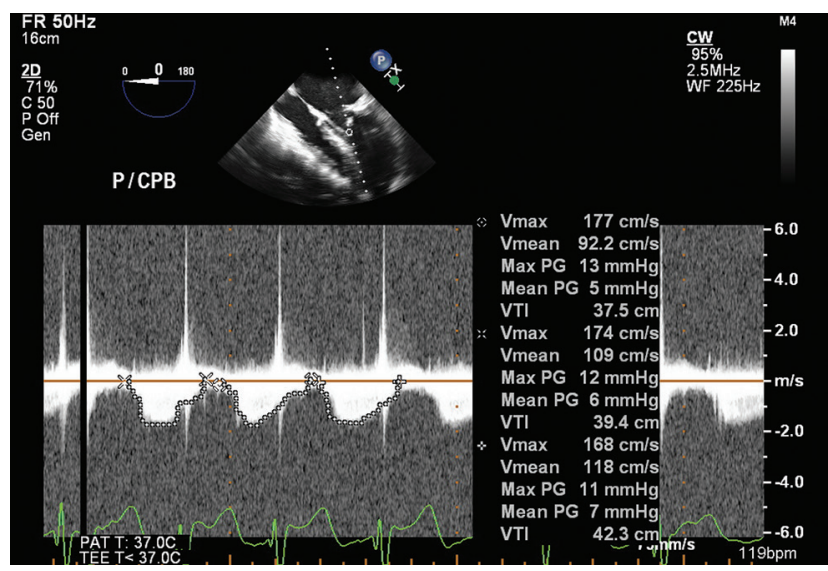

Fig. 6 Two-dimensional midesophageal four chamber transesophageal echocardiographic view at 0 degrees post first cardiopulmonary bypass, showing average mean gradients of three beats across mitral valve of $6 \mathrm{~mm} \mathrm{Hg}$.

\section{Discussion}

Various prospective and retrospective studies advocate better long-term outcome for patients with mitral valve repair with concomitant aortic valve replacement over double valve replacement. ${ }^{1-3}$ McGonigle et al in a retrospective analysis confirmed significant benefit in survival postsurgery, following either technique. ${ }^{4}$ Postoperative complications like bleeding, reoperations, infective endocarditis, and thromboembolic events have similar incidence among the two surgical techniques. However, the survival rates are significantly better in the population undergoing mitral valve repair with aortic valve replacement compared with double valve replacement patients. Better prognosis postsurgery is attributed to preserved native valve and requirements of lesser International Normalized Ratio (INR) postmitral valve repair (INR 2-2.5) compared with patients with double prosthetic valves (INR 2.5-3.5). ${ }^{5}$

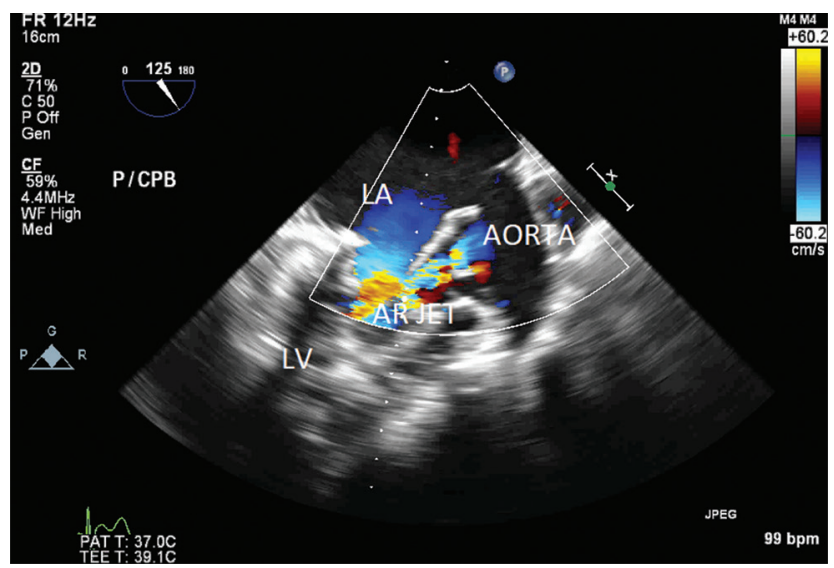

Fig. 7 Two-dimensional midesophageal aortic valve long axis transesophageal echocardiographic view at 125 degrees, showing moderate residual regurgitant jet across aortic valve. Abbreviations: LA, left atrium; LV, left ventricle; AR, aortic regurgitation.

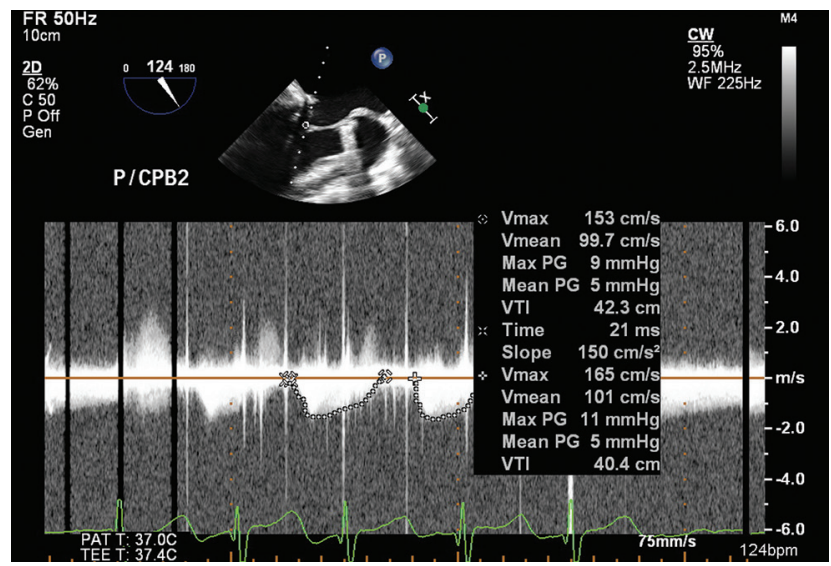

Fig. 8 Two-dimensional midesophageal long axis transesophageal echocardiographic view at 124 degrees,, showing average of two beats mean mitral valve gradient of $5 \mathrm{~mm} \mathrm{Hg}$.

Our patient being on ART itself challenges postoperative care. Drug interactions associated with ART, as well as increased predisposition to infections and increased requirement of coagulation monitoring, significantly impacts postoperative care and dosing of anticoagulants. Induction or inhibition of warfarin metabolism through CYP2C9 may occur, depending on the specific antiretroviral agent, especially when protein inhibitors (PIs) or non-nucleoside reverse transcriptase inhibitors (NNRTIs) are used. ${ }^{6}$ To achieve therapeutic INR levels, approximately 50\% more warfarin is required in patients on either lopinavir/ ritonavir or atazanavir/ritonavir-based regimens with NNRTIs compared with patients on efavirenz with NNRTIs. Neither of the new oral anticoagulants like Dabigatran, a direct thrombin inhibitor, and rivaroxaban, a factor Xa inhibitor, require the frequent monitoring of warfarin; and dabigatran in particular appears to have fewer interactions with antiretrovirals. These agents are a better future prospective for individuals who require long-term anticoagulation. 
Several caveats need to be closely considered while discussing the pertinent issue of a sound decision-making in such a clinical setting. First, albeit the fact that there are several factors eulogizing the peculiar advantages attributable to valvular repairs, reparative valvular surgery continues to be a laggard in the developing world. In addition, to the clarion call of disseminating reparative techniques, the preponderance of rheumatic etiology in our setup in contrast to the rather conducive degenerative variety complicates the situation furthermore in the developed world. Second, preoperative TEE valvular disease severity examination is compounded by the alterations in the filling pressures, owing to multivalvular pathology. Therefore, the role of postoperative TEE in assessing the adequacy of repair cannot be overemphasized. Third, "repair/replace" decisions need to be formulated in a holistic manner, accounting for the patient profile, as elucidated in the present case.

\section{Conclusion}

The aforementioned discussion presents a nuanced perspective on surgical decision-making in multivalvular heart disease, wherein an effective communication between the surgeon and the echocardiographer for ensuring favorable postoperative outcomes often yields valuable clues to the process of intraoperative decision formulation.

\section{Conflicts of Interest}

None.

\section{References}

1 Hamamoto M, Bando K, Kobayashi J, et al. Durability and outcome of aortic valve replacement with mitral valve repair versus double valve replacement. Ann Thorac Surg 2003;75(1):28-33, discussion 33-34

2 Kim GS, Kim JB, Han S, et al. Mitral valve repair versus replacement for moderate-to-severe mitral regurgitation in patients undergoing concomitant aortic valve replacement. Interact Cardiovasc Thorac Surg 2014;18(1):73-79

3 Vohra HA, Whistance RN, Hechadi J, et al. Long-term outcomes of concomitant aortic and mitral valve repair. J Thorac Cardiovasc Surg 2014;148(2):454-460

4 McGonigle NC, Jones JM, Sidhu P, Macgowan SW. Concomitant mitral valve surgery with aortic valve replacement: a 21-year experience with a single mechanical prosthesis. J Cardiothorac Surg 2007;2-24

5 Nishimura RA, Otto CM, Bonow RO, et al. 2017 AHA/ACC Focused Update of the 2014 AHA/ACC Guideline for the Management of Patients With Valvular Heart Disease: a report of the American College of Cardiology/American Heart Association Task Force on Clinical Practice Guidelines. J Am Coll Cardiol 2017;70(2):252-289

6 Liedtke MD, Rathbun RC. Warfarin-antiretroviral interactions. Ann Pharmacother 2009;43(2):322-328

7 Anderson AM, Chane T, Patel M, Chen S, Xue W, Easley KA. Warfarin therapy in the HIV medical home model: low rates of therapeutic anticoagulation despite adherence and differences in dosing based on specific antiretrovirals. AIDS Patient Care STDS 2012;26(8):454-462 